\title{
NONAUTONOMOUS CONLEY INDEX THEORY THE CONNECTING HOMOMORPHISM
}

\author{
AXEL JÄNIG
}

\begin{abstract}
Attractor-repeller decompositions of isolated invariant sets give rise to so-called connecting homomorphisms. These homomorphisms reveal information on the existence and structure of connecting trajectories of the underlying dynamical system.

To give a meaningful generalization of this general principle to nonautonomous problems, the nonautonomous homology Conley index is expressed as a direct limit. Moreover, it is shown that a nontrivial connecting homomorphism implies, on the dynamical systems level, a sort of uniform connectedness of the attractor-repeller decomposition.
\end{abstract}

In previous works [6, 7] the author developed a nonautonomous Conley index theory. The index relies on the interplay between a skew-product semiflow and a nonautonomous evolution operator. It can be applied to various nonautonomous problems, including ordinary differential equations and semilinear parabolic equations (see [6]).

Every attractor-repeller decomposition of an isolated invariant set gives rise to a long exact sequence involving the homology Conley index. The connecting homomorphism of this sequence contains information on the connections between repeller and attractor. In particular, the connecting homomorphism vanishes if a connecting orbit does not exist.

However, the mere existence of a connecting orbit is a very weak result in the context of nonautonomous dynamical systems. This is due to the fact that, in general, a nonautonomous Conley index is always tied to a family of nonautonomous dynamical systems. Therefore, for a specific dynamical system, only (in an appropriate sense) uniform properties are meaningful. It will be shown that the existence of connections due to a non-trivial connecting homomorphism is such a uniform property.

More precisely, the nonautonomous homology Conley index is expressed as a direct limit, which resembles the definition of a discrete Conley index. Using the direct limit formula, a notion of uniform connectedness of the attractor-repeller decomposition respectively the invariant set is introduced. Moreover, it is shown that a non trivial connecting homomorphism implies not only the existence of a connection but a uniform connection of repeller and attractor.

2010 Mathematics Subject Classification. Primary: 37B30, 37B55; Secondary: 34C99, 35B08, 35B40.

Key words and phrases. nonautonomous differential equations, attractor-repeller decompositions, connecting orbits, connecting homomorphism, perturbations, semilinear parabolic equations, Morse-Conley index theory, partially orderd Morse-decompositions, nonautonomous Conley index, homology Conley index. 
Theorem 1.1 below, included mainly for illustrative purposes, translates the results of this paper to the problem of small perturbations of a class of semilinear parabolic equations e.g., reaction diffusion equations. The main part of the paper starts with a Preliminaries section, where we collect important definitions and results from other works. In Section 3, the direct limit formulation is formulated and proved. The notion of uniform connectedness is introduced in Section 4. Subsequently, we prove Theorem 4.2 stating that a nontrivial connecting homomorphism implies a uniform connection of repeller and attractor.

\section{Nonautonomous $C^{0}$-Small perturbations of (AUtonomous) SEMILINEAR PARABOLIC EQUATIONS}

Let $X$ be a Banach space and $A_{0}$ be a sectorial operator defined on a dense subset $\mathcal{D}\left(A_{0}\right) \subset X$. We are interested in mild solutions of

$$
u_{t}+A_{0} u=\hat{f}(t, u)
$$

which happen to be strong solutions due to regularity assumptions. Let us further assume that $A_{0}$ has compact resolvent.

As often, the operator $A_{0}$ is assumed to be positive, so there is a family of fractional power spaces $X^{\alpha}$ defined by $A_{0}$. The respective norm is given by $\|x\|_{\alpha}:=$ $\left\|A_{0}^{\alpha} x\right\|_{X}$. A typical example would be the Laplace-operator on a bounded domain with smooth boundary under appropriate boundary conditions (see e.g. [3, 9, 11]).

We will shortly introduce another metric space $Y$. With every $f \in Y$ there is an associated mapping $\hat{f}$, which serves a a parameter for the evolution operator defined by (1). A typical example for $\hat{f}$ is assigning the Nemitskii operator associated with a function $f$.

We are interested $C^{0}$-small nonautonomous perturbations of autonomous equations that is,

$$
u_{t}+A_{0} u=\hat{f}(u)+\hat{g}(t, u)
$$

where $g$ is assumed to be small in an appropriate metric. Note that this is much stronger than

$$
u_{t}+A_{0} u=\hat{f}(u)+\varepsilon \hat{g}(t, u)
$$

where $\varepsilon$ is assumed to be small.

The main result is the persistence of Morse-decompositions and certain solutions: Morse-sets with a non-zero index as well as connecting orbits with a non-vanishing connecting homomorphism. A typical Morse-set with non-zero index might be a hyperbolic equilibrium and a typical Morse-set with a non-vanishing connecting homomorphism might be a transversal heteroclinic solution (see [5]).

In the sequel, a specific choice for $Y$ is made. Additional material can be found in [6] and [12].

Let $\Omega \subset \mathbb{R}^{N}$ be a smooth bounded domain, and suppose that, for some $\alpha \in[0,1[$, there is a continuous inclusion $X^{\alpha} \subset C(\bar{\Omega})$. Let $Y$ denote the set of all continuous functions $f: \mathbb{R} \times \bar{\Omega} \times \mathbb{R} \rightarrow \mathbb{R}$ which are subject of the following restriction:

For some $\delta>0$ and every $C_{1}>0$, there are constants $C_{2}=C_{2}\left(C_{1}\right)$ and $C_{3}=C_{3}\left(C_{1}\right)$ such that for all $(t, x, u) \in \mathbb{R} \times \Omega \times \mathbb{R}$ with $|u| \leq C_{1}$

$$
|f(t, x, u)| \leq C_{2}
$$




$$
\begin{gathered}
\text { and for all }\left(t_{1}, x_{1}, u_{1}\right),\left(t_{2}, x_{2}, u_{2}\right) \in \mathbb{R} \times \Omega \times \mathbb{R} \text { with }\left|u_{1}\right|,\left|u_{1}\right| \leq C_{1} \\
\left|f\left(t_{1}, x_{1}, u_{1}\right)-f\left(t_{2}, x_{2}, u_{2}\right)\right| \leq C_{3}\left(\left|t_{1}-t_{2}\right|^{\delta}+\left|x_{1}-x_{2}\right|^{\delta}+\left|u_{1}-u_{2}\right|\right)
\end{gathered}
$$

Defining addition and scalar multiplication pointwise as usual, $Y$ becomes a linear space. We consider a family $\left(\delta_{n}\right)_{n \in \mathbb{N}}$ of seminorms

$$
\delta_{n}(f):=\sup \{|f(t, x, u)|:(t, x, u) \in \mathbb{R} \times \Omega \times \mathbb{R} \text { with }|t|,|u| \leq n\} .
$$

These seminorms give rise to an invariant metric $d$ on $Y$ :

$$
d\left(f_{1}, f_{2}\right):=\sum_{n=1}^{\infty} 2^{-n} \frac{\delta_{n}\left(f_{1}-f_{2}\right)}{1+\delta_{n}\left(f_{1}-f_{2}\right)} .
$$

The metric $d$ induces the compact-open topology on $Y$, so a sequence of functions converges with respect to $d$ if and only if it converges uniformly on bounded subsets of $\mathbb{R} \times \Omega \times \mathbb{R}$.

To formulate the theorem below, a uniform distance is also required i.e.,

$$
d_{\text {unif }}\left(f_{1}, f_{2}\right):=\sup _{(t, x, u) \in \mathbb{R} \times \Omega \times \mathbb{R}}\left|f_{1}(t, x, u)-f_{2}(t, x, u)\right|
$$

Denote

$$
h: \mathbb{R} \rightarrow \mathbb{R} \quad h(t):= \begin{cases}(t+1) \sin \ln (t+1) & t>0 \\ 0 & t \leq 0\end{cases}
$$

and $t_{n}:=e^{2 \pi n}-1, n \in \mathbb{N}$. Then, $\ln \left(t_{n}+s\right)-2 \pi n \rightarrow 0$ as $n \rightarrow \infty$ uniformly for $s$ lying in bounded subsets of $\mathbb{R}$. Hence, one has

$$
h\left(t_{n}+s\right)=h\left(t_{n}\right)+\int_{t_{n}}^{t_{n}+s} \sin \ln (t+1)+\cos \ln (t+1) \mathrm{d} t
$$

so that $h\left(t_{n}+s\right) \rightarrow s$ as $n \rightarrow \infty$ uniformly on bounded sets.

We are interested in full (defined on $\mathbb{R}$ ) solutions of a perturbed equation. Suppose that $f \in Y$ is the parameter associated with the perturbed equation. Computing the index with respect to $f$ would imply the loss of all the information contained in $f$ for negative times. The index would be determined by the equation's behaviour at large times. This restriction can be overcome by using the auxiliary function $h$ defined above. It allows to embed $f$ into the $\omega$-limes set of a related parameter, namely:

$$
f . h:=((t, x, u) \mapsto f(h(t), x, u)) .
$$

It is easy to see that $f \in Y$ implies $f . h \in Y$ and, from the calculations above, it follows that $(f . h)^{t_{n}} \rightarrow f$ in $Y$, that is, uniformly on bounded subsets.

Combining this approach with the abstract results of this paper and previous works on the subject, one obtains the following theorem.

Theorem 1.1. Suppose that $f \in Y$ is autonomous, and let $K \subset X^{\alpha}$ be a compact invariant set with respect to the evolution operator (semiflow) on $X^{\alpha}$ defined by (11). Let $N \subset X^{\alpha}$ be a strongly admissible (e.g. bounded) isolated neighbourhood of $K$.

Let $(A, R)$ be an attractor-repeller decomposition of $K$, and assume that the associated connecting homomorphism $\partial: \mathrm{H}_{*} \mathcal{C}(f, A) \rightarrow \mathrm{H}_{*} \mathcal{C}(f, R)$ defined by the homology attractor-repeller sequence does not vanish. 
Let $N_{A} \subset X^{\alpha}$ (resp. $N_{R}$ ) be an isolating neighbourhood for $A$ (resp. $R$ ), and suppose that $N_{A} \cap N_{R}=\emptyset$.

Then, there exists an $\varepsilon>0$ such that the following holds true for all $f^{\prime} \in Y$ with $d_{\text {unif }}\left(f, f^{\prime}\right)<\varepsilon$ :

(a) If $u: \mathbb{R} \rightarrow N \subset X^{\alpha}$ is a solution of

$$
u_{t}+A_{0} u=\hat{f}^{\prime}(t, u),
$$

then either $u(\mathbb{R}) \subset N_{A} \cup N_{R}$ or $\alpha(u) \subset N_{R}$ and $\omega(u) \subset N_{A}$.

(b) There is a solution $u: \mathbb{R} \rightarrow N_{R}$ of (2).

(c) There is a solution $u: \mathbb{R} \rightarrow N_{A}$ of (2).

(d) Suppose $U_{A} \subset X^{\alpha}$ is a neighbourhood of $A, U_{R} \subset X^{\alpha}$ a neighbourhood of $R$ and $U_{A} \cap U_{A}=\emptyset$. Then there is a solution $u: \mathbb{R} \rightarrow N$ of (2) such that $\alpha(u) \subset N_{R}, \omega(u) \subset N_{A}$ and $u(0) \in X^{\alpha} \backslash\left(U_{A} \cup U_{R}\right)$.

Using the same arguments as below, the theorem can be generalized to partially ordered Morse decompositions (see 4]). Moreover, even if the connecting homomorphism is tribal, Morse-decompositions are still preserved under small perturbations, but depending on the Conley indices of attractor and repeller, the existence of solutions can no longer be proved].

Note that for a generic reaction diffusion equation, all equilibria are hyperbolic, and their respective stable and unstable manifolds intersect transversally [1]. For such a generic reaction-diffusion equation each heteroclinic connection between equilibria of adjacent Morse indices corresponds to a nontrivial connecting homomorphism [5].

Proof. First of all, note that $f . h=f$ as $f$ is autonomous. Furthermore, $Y \times N$, $Y \times N_{A}$ and $Y \times N_{R}$ are isolating neighbourhoods for $(f . h, K),(f . h, A)$ and $(f . h, R)$ respectively.

Therefore, (a) follows from Theorem 2.2 in 4 . We will now consider (the relevant part of) an attractor-repeller sequence containing the non-vanishing connecting homomorphism $\partial$ :

$$
\longrightarrow \mathrm{H}_{*} \mathcal{C}(f, R) \stackrel{\partial}{\longrightarrow} \mathrm{H}_{*-1} \mathcal{C}(f, A) \longrightarrow
$$

Since $\partial \neq 0$, one necessarily has $\mathrm{H}_{*} \mathcal{C}(f, R) \neq 0$ and $\mathrm{H}_{*} \mathcal{C}(f, A) \neq 0$. By using Theorem 3.4 in [4, one proves that for all $f^{\prime}$ in a neighbourhood of $f$ given by (a), the attractor-repeller sequence above extends to a commutative ladder:

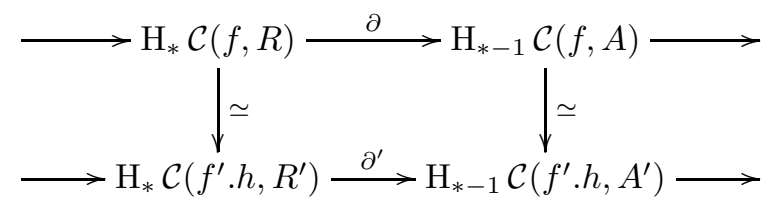

Here, we set $R^{\prime}:=\operatorname{Inv}\left(\omega\left(f^{\prime} . h\right) \times N_{R}\right)$ and $A^{\prime}:=\operatorname{Inv}\left(\omega\left(f^{\prime} . h\right) \times N_{A}\right)$.

Consequently, in view of Corollary 4.11 in [6] and because $f^{\prime} \in \omega\left(f^{\prime} . h\right)$, (b) and (c) must hold. Finally, claim (d) is a consequence of Theorem 4.2 stating that $K^{\prime}=\operatorname{Inv}\left(\omega\left(f^{\prime} . h\right) \times N\right)$ is uniformly connected.

\footnotetext{
${ }^{1}$ At least not with these arguments.
} 


\section{Preliminaries}

The section starts with a collection of useful definitions and terminology, mainly from [6] and [7]. Thereafter, we review the concept of index pairs and index triples, followed by the nonautonomous homology Conley index and the connecting homomorphism.

\subsection{Quotient spaces.}

Definition 2.1. Let $X$ be a topological space, and $A, B \subset X$. Denote

$$
A / B:=A / R \cup\{A \cap B\},
$$

where $A / R$ is the set of equivalence classes with respect to the relation $R$ on $A$ which is defined by $x R y$ iff $x=y$ or $x, y \in B$.

We consider $A / B$ as a topological space endowed with the quotient topology with respect to the canonical projection $q: A \rightarrow A / B$, that is, a set $U \subset A / B$ is open if and only if

$$
q^{-1}(U)=\bigcup_{x \in U} x
$$

is open in $A$.

Recall that the quotient topology is the final topology with respect to the projection $q$.

Remark 2.2. The above definition is compatible with the definition used in [2] or [10. The only difference occurs in the case $A \cap B=\emptyset$, where we add $\emptyset$, which is never an equivalence class, instead of an arbitrary point.

2.2. Evolution operators and semiflows. Let $X$ be a metric space. Assuming that $\diamond \notin X$, we introduce a symbol $\diamond$, which means "undefined". The intention is to avoid the distinction if an evolution operator is defined for a given argument or not. Define $\bar{A}:=A \dot{\cup}\{\diamond\}$ whenever $A$ is a set with $\diamond \notin A$. Note that $\bar{A}$ is merely a set, the notation does not contain any implicit assumption on the topology.

Definition 2.3. Let $\Delta:=\left\{\left(t, t_{0}\right) \in \mathbb{R}^{+} \times \mathbb{R}^{+}: t \geq t_{0}\right\}$. A mapping $\Phi: \Delta \times \bar{X} \rightarrow \bar{X}$ is called an evolution operator if

(1) $\mathcal{D}(\Phi):=\left\{\left(t, t_{0}, x\right) \in \Delta \times X: \Phi\left(t, t_{0}, x\right) \neq \diamond\right\}$ is open in $\mathbb{R}^{+} \times \mathbb{R}^{+} \times X$;

(2) $\Phi$ is continuous on $\mathcal{D}(\Phi)$;

(3) $\Phi\left(t_{0}, t_{0}, x\right)=x$ for all $\left(t_{0}, x\right) \in \mathbb{R}^{+} \times X$;

(4) $\Phi\left(t_{2}, t_{0}, x\right)=\Phi\left(t_{2}, t_{1}, \Phi\left(t_{1}, t_{0}, x\right)\right)$ for all $t_{0} \leq t_{1} \leq t_{2}$ in $\mathbb{R}^{+}$and $x \in X$;

(5) $\Phi\left(t, t_{0}, \diamond\right)=\diamond$ for all $t \geq t_{0}$ in $\mathbb{R}^{+}$.

A mapping $\pi: \mathbb{R}^{+} \times \bar{X} \rightarrow \bar{X}$ is called semiflow if $\tilde{\Phi}\left(t+t_{0}, t_{0}, x\right):=\pi(t, x)$ defines an evolution operator. To every evolution operator $\Phi$, there is an associated (skewproduct) semiflow $\pi$ on an extended phase space $\mathbb{R}^{+} \times X$, defined by $\left(t_{0}, x\right) \pi t=$ $\left(t_{0}+t, \Phi\left(t+t_{0}, t_{0}, x\right)\right)$.

A function $u: I \rightarrow X$ defined on a subinterval $I$ of $\mathbb{R}$ is called a solution of (with respect to) $\Phi$ if $u\left(t_{1}\right)=\Phi\left(t_{1}, t_{0}, u\left(t_{0}\right)\right)$ for all $\left[t_{0}, t_{1}\right] \subset I$.

Definition 2.4. Let $X$ be a metric space, $N \subset X$ and $\pi$ a semiflow on $X$. The set

$$
\operatorname{Inv}_{\pi}^{-}(N):=\left\{x \in N: \text { there is a solution } u: \mathbb{R}^{-} \rightarrow N \text { with } u(0)=x\right\}
$$

is called the largest negatively invariant subset of $N$. 
The set

$$
\operatorname{Inv}_{\pi}^{+}(N):=\left\{x \in N: x \pi \mathbb{R}^{+} \subset N\right\}
$$

is called the largest positively invariant subset of $N$.

The set

$$
\operatorname{Inv}_{\pi}(N):=\{x \in N: \text { there is a solution } u: \mathbb{R} \rightarrow N \text { with } u(0)=x\}
$$

is called the largest invariant subset of $N$.

Let $X$ and $Y$ be metric spaces, and assume that $y \mapsto y^{t}$ is a global2 semiflow on $Y$, to which we will refer as $t$-translation.

Example 2.5. Let $Z$ be a metric space, and let $Y:=C\left(\mathbb{R}^{+}, Z\right)$ be a metric space such that a sequence of functions converges if and only if it converges uniformly on bounded sets. The translation can now be defined canonically by $y^{t}(s):=y(t+s)$ for $s, t \in \mathbb{R}^{+}$.

A suitable abstraction of many non-autonomous problems is given by the concept of skew-product semiflows introduced below.

Definition 2.6. We say that $\pi=\left(.^{t}, \Phi\right)$ is a skew-product semiflow on $Y \times X$ if $\Phi: \mathbb{R}^{+} \times \overline{Y \times X} \rightarrow \overline{Y \times X}$ is a mapping such that

$$
(t, y, x) \pi t:= \begin{cases}\left(y^{t}, \Phi(t, y, x)\right) & \Phi(t, y, x) \neq \diamond \\ \diamond & \text { otherwise }\end{cases}
$$

is a semiflow on $Y \times X$.

A skew-product semiflow gives rise to evolution operators.

Definition 2.7. Let $\pi=\left(.^{t}, \Phi\right)$ be a skew-product semiflow and $y \in Y$. Define

$$
\Phi_{y}\left(t+t_{0}, t_{0}, x\right):=\Phi\left(t, y^{t_{0}}, x\right) .
$$

It is easily proved that $\Phi_{y}$ is an evolution operator in the sense of Definition 2.3 ,

Definition 2.8. For $y \in Y$ let

$$
\mathcal{H}^{+}(y):=\operatorname{cl}_{Y}\left\{y^{t}: t \in \mathbb{R}^{+}\right\}
$$

denote the positive hull of $y$. Let $Y_{c}$ denote the set of all $y \in Y$ for which $\mathcal{H}^{+}(y)$ is compact.

Definition 2.9. Let $y_{0} \in Y$ and $K \subset \mathcal{H}^{+}\left(y_{0}\right) \times X$ be an invariant set. A closed set $N \subset Y \times X$ (resp. $\left.N \subset \mathcal{H}^{+}\left(y_{0}\right) \times X\right)$ is called an isolating neighbourhood for $\left(y_{0}, K\right)($ in $Y \times X)\left(\right.$ resp. in $\left.\mathcal{H}^{+}\left(y_{0}\right) \times X\right)$ provided that:

(1) $K \subset \mathcal{H}^{+}\left(y_{0}\right) \times X$

(2) $K \subset \operatorname{int}_{Y \times X} N$ (resp. $\left.K \subset \operatorname{int}_{\mathcal{H}^{+}\left(y_{0}\right) \times X} N\right)$

(3) $K$ is the largest invariant subset of $N \cap\left(\mathcal{H}^{+}\left(y_{0}\right) \times X\right)$

The following definition is a consequence of the slightly modified notion of a semiflow (Definition 2.3) but not a semantic change compared to [2, for instance.

Definition 2.10. We say that $\pi$ explodes in $N \subset Y \times X$ if $x \pi[0, t[\subset N$ and $x \pi t=\diamond$.

Following [10, we formulate the following asymptotic compactness condition.

\footnotetext{
${ }^{2}$ defined for all $t \in \mathbb{R}^{+}$
} 
Definition 2.11. A set $M \subset Y \times X$ is called strongly admissible provided the following holds:

Whenever $\left(y_{n}, x_{n}\right)$ is a sequence in $M$ and $\left(t_{n}\right)_{n}$ is a sequence in $\mathbb{R}^{+}$such that $\left(y_{n}, x_{n}\right) \pi\left[0, t_{n}\right] \subset M$, then the sequence $\left(y_{n}, x_{n}\right) \pi t_{n}$ has a convergent subsequence.

2.3. Index pairs and index triples. The notion of (basic) index pairs relies on [6] and was introduced in 7 .

Definition 2.12. A pair $\left(N_{1}, N_{2}\right)$ is called a (basic) index pair relative to a semiflow $\chi$ in $\mathbb{R}^{+} \times X$ if

(IP1) $N_{2} \subset N_{1} \subset \mathbb{R}^{+} \times X, N_{1}$ and $N_{2}$ are closed in $\mathbb{R}^{+} \times X$

(IP2) If $x \in N_{1}$ and $x \chi t \notin N_{1}$ for some $t \in \mathbb{R}^{+}$, then $x \chi s \in N_{2}$ for some $s \in[0, t]$;

(IP3) If $x \in N_{2}$ and $x \chi t \notin N_{2}$ for some $t \in \mathbb{R}^{+}$, then $x \chi s \in\left(\mathbb{R}^{+} \times X\right) \backslash N_{1}$ for some $s \in[0, t]$.

Definition 2.13. Let $y_{0} \in Y$ and $\left(N_{1}, N_{2}\right)$ be a basic index pair in $\mathbb{R}^{+} \times X$ relative to $\chi_{y_{0}}$. Define $r:=r_{y_{0}}: \mathbb{R}^{+} \times X \rightarrow \mathcal{H}^{+}\left(y_{0}\right) \times X$ by $r_{y_{0}}(t, x):=\left(y_{0}^{t}, x\right)$.

Let $K \subset \omega\left(y_{0}\right) \times X$ be an (isolated) invariant set. We say that $\left(N_{1}, N_{2}\right)$ is a (strongly admissible) index pain 3 for $\left(y_{0}, K\right)$ if:

(IP4) there is a strongly admissible isolating neighborhood $N$ of $K$ in $\mathcal{H}^{+}\left(y_{0}\right) \times X$ such that $N_{1} \backslash N_{2} \subset r^{-1}(N)$;

(IP5) there is a neighbourhood $W$ of $K$ in $\mathcal{H}^{+}\left(y_{0}\right) \times X$ such that $r^{-1}(W) \subset$ $N_{1} \backslash N_{2}$.

Definition 2.14. Let $\left(N_{1}, N_{2}\right)$ be an index pair in $\mathbb{R}^{+} \times X$ (relative to the semiflow $\chi$ on $\left.\mathbb{R}^{+} \times X\right)$. For $T \in \mathbb{R}^{+}$, we set

$$
N_{2}^{-T}:=N_{2}^{-T}\left(N_{1}\right):=\left\{(t, x) \in N_{1}: \exists s \leq T(t, x) \chi s \in N_{2}\right\}
$$

To define index triples, the notion of an attractor-repeller decomposition is required.

First of all, $\alpha$ and $\omega$-limes sets can be defined as usual.

$$
\begin{aligned}
\alpha(u) & \left.\left.:=\bigcap_{t \in \mathbb{R}^{-}} \operatorname{cl}_{\mathcal{H}^{+}\left(y_{0}\right) \times X} u(]-\infty, t\right]\right) \\
\omega(u) & :=\bigcap_{t \in \mathbb{R}^{+}} \operatorname{cl}_{\mathcal{H}^{+}\left(y_{0}\right) \times X} u([t, \infty[)
\end{aligned}
$$

Based on these definitions, the notion of an attractor-repeller decomposition can be made precise.

Definition 2.15. Let $y_{0} \in Y$ and $K \subset \mathcal{H}^{+}\left(y_{0}\right) \times X$ be an isolated invariant set. $(A, R)$ is an attractor-repeller decomposition of $K$ if $A, R$ are disjoint isolated invariant subsets of $K$ and for every solution $u: \mathbb{R} \rightarrow K$ one of the following alternatives holds true.

(1) $u(\mathbb{R}) \subset A$

(2) $u(\mathbb{R}) \subset R$

(3) $\alpha(u) \subset R$ and $\omega(u) \subset A$

We also say that $\left(y_{0}, K, A, R\right)$ is an attractor-repeller decomposition.

Finally, index triples (which correspond to attractor-repeller decompositions) are defined.

\footnotetext{
${ }^{3}$ Every index pair in the sense of Definition 2.13 is assumed to be strongly admissible.
} 
Definition 2.16. Let $y_{0} \in Y$ and $K \subset \mathcal{H}^{+}\left(y_{0}\right) \times X$ be an isolated invariant set admitting a strongly admissible isolating neighbourhood $N$. Suppose that $(A, R)$ is an attractor-repeller decomposition of $K$.

A triple $\left(N_{1}, N_{2}, N_{3}\right)$ is called an index triple for $\left(y_{0}, K, A, R\right)$ provided that:

(1) $N_{3} \subset N_{2} \subset N_{1}$

(2) $\left(N_{1}, N_{3}\right)$ is an index pair for $\left(y_{0}, K\right)$

(3) $\left(N_{2}, N_{3}\right)$ is an index pair for $\left(y_{0}, A\right)$

2.4. Homology Conley index and attractor repeller decompositions. A connected simple system is a small category such that given a pair $(A, B)$ of objects, there is exactly one morphism $A \rightarrow B$.

Let $y_{0} \in Y_{c}$ and $K \subset \mathcal{H}^{+}\left(y_{0}\right) \times X$ be an isolated invariant set for which there is a strongly admissible isolating neighbourhood. The categorial Conley index $\mathcal{C}\left(y_{0}, K\right)$ (as defined in [7]) is a subcategory of the homotopy category of pointed spaces and a connected simple system. Its objects are the index pairs for $\left(y_{0}, K\right)$. Roughly speaking, one can think of an index pair with collapsed exit set as a representative of the index. All of the representatives are isomorphic in the homotopy category of pointed spaces.

Let $\left(\mathrm{H}_{*}, \partial\right)$ denote a homology theory with compact supports [13]. Recall that $\mathrm{H}_{*}$ is a covariant function from the category of topological pairs to the category of graded abelian groups (or modules).

Define the homology Conley index $\mathrm{H}_{*} \mathcal{C}\left(y_{0}, K\right)$ to be the following connected simple system: $\mathrm{H}_{*}\left(N_{1} / N_{2},\left\{N_{2}\right\}\right)$ is an object whenever $\left(N_{1} / N_{2}, N_{2}\right)$ is an object of $\mathcal{C}\left(y_{0}, K\right)$. The morphisms of $\mathrm{H}_{*} \mathcal{C}\left(y_{0}, K\right)$ are obtained analogously from the morphisms of $\mathcal{C}\left(y_{0}, K\right)$. Note that we also write $\mathrm{H}_{*}\left(A, a_{0}\right):=\mathrm{H}_{*}\left(A,\left\{a_{0}\right\}\right)$ provided the meaning is clear.

Let $\left(y_{0}, K, A, R\right)$ be an attractor-repeller decomposition. There is a long exact [7] sequence

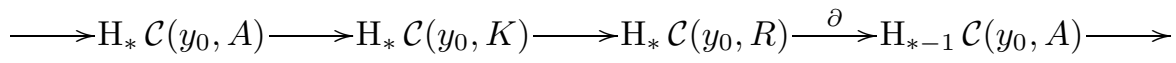

where $\partial$ denotes the connecting homomorphism.

\section{The homology Conley indeX as a DiReCt limit}

Let $\left(N_{1}, N_{2}\right)$ be a basic index pair. Another basic index pair is $\left(N_{1}([t, \infty[)\right.$, $N_{2}\left([t, \infty[))\right.$, where $t>0$ is arbitrary and $N_{i}\left(\left[t, \infty[)=\left\{(s, x) \in N_{i}: s \geq t\right\}\right.\right.$ for $i \in\{1,2\}$. The inclusion $\left(N_{1}\left(\left[t, \infty[), N_{2}\left([t, \infty[)) \subset\left(N_{1}, N_{2}\right)\right.\right.\right.\right.$ induces a homotopy equivalence between the (pointed) quotient spaces $\left(N_{1}\left(\left[t, \infty[) / N_{2}([t, \infty[)\right.\right.\right.$, $N_{2}\left([t, \infty[))\right.$ and $\left(N_{1} / N_{2}, N_{2}\right)$. Apparently, only the index pair at large times is relevant.

In the present section, this limit behaviour will be studied. Finite sections of an index pair $\left(N_{1}, N_{2}\right)$, that is, sets of the form $N_{i}[\alpha, \beta]=N_{i} \cap([\alpha, \beta] \times X)$, in conjunction with appropriate morphisms form a direct system. The index $\mathrm{H}_{*}\left(N_{1} / N_{2}, N_{2} / N_{2}\right)$ is then proved to be isomorphic to a direct limit obtained from these sections.

It is interesting to note that this result (in particular Lemma 3.8) resembles constructing a Conley index for discrete time dynamical systems (see e.g. 8]). In this paper, however, we will focus on the use of the direct limit representation of the index as a tool. 
For the rest of this section, let $\Lambda$ be a set and $\leq$ a partial order on $\Lambda$. Recall [13] that a direct system of sets is a family $\left(A_{\alpha}\right)_{\alpha \in \Lambda}$ of sets and a family of functions $\left(f_{\alpha, \beta}\right)$, where $\alpha, \beta \in \Lambda, \alpha \leq \beta$ and $f_{\alpha, \beta}: A_{\alpha} \rightarrow A_{\beta}$.

The direct limit $\operatorname{dirlim}\left(A_{\alpha}, f_{\alpha, \beta}\right)$ of $\left(A_{\alpha}, f_{\alpha, \beta}\right)$ is the set of equivalence classes in $\bigcup_{\alpha \in \Lambda}\{\alpha\} \times A_{\alpha}$ under the relation $\sim$, which is defined as follows: Let $\alpha, \beta \in \Lambda$ and $(a, b) \in A^{\alpha} \times A^{\beta} .(\alpha, a) \sim(\beta, b)$ if and only if there is a $\gamma \in \Lambda$ such that $\alpha, \beta \leq \gamma$ and $f_{\alpha, \gamma}(a)=f_{\beta, \gamma}(b)$.

Let $(X, d)$ be a complete metric space, and $V \subset \mathbb{R}^{+} \times X$. We set

$$
\begin{aligned}
V(t) & :=\{x:(t, x) \in V\} \\
V([a, b]):=V[a, b] & :=\{(t, x) \in V: t \in[a, b]\} .
\end{aligned}
$$

Definition 3.1. An index pair $\left(N_{1}, N_{2}\right)$ is called regular (with respect to $y_{0}$ ) if the (inner) exit time $T_{i}: N_{1} \rightarrow[0, \infty], T_{i}(x):=\sup \left\{t \in \mathbb{R}^{+}: x \chi_{y_{0}}[0, t] \subset N_{1} \backslash N_{2}\right\}$ is continuous.

The main motivation for regular index pairs are Lemma 3.2 below and Lemma 3.8 at the end of this section. As stated subsequently in Lemma 3.3, it is easily possible to obtain regular index pairs by modifying (enlarging) the exit set appropriately. The following notational shortcut is used frequently.

$$
\mathrm{H}_{*}[A, B]:=\mathrm{H}_{*}(A / B,\{B\})
$$

Lemma 3.2. Let $\left(N_{1}, N_{2}\right)$ be a regular index pair in $\mathbb{R}^{+} \times X$.

Consider the direct system $\left(A_{\alpha}, f_{\alpha, \beta}\right)$ for $\alpha, \beta \in \Lambda$, where $\Lambda$ denotes the set of all non-empty compact subintervals of $\mathbb{R}^{+}$ordered by inclusion, and $A_{\alpha}:=$ $H_{*}\left[N_{1}(\alpha), N_{2}(\alpha)\right]$. For $\alpha \subset \beta$, let $i_{\alpha, \beta}:\left(N_{1}(\alpha), N_{2}(\alpha)\right) \rightarrow\left(N_{1}(\beta), N_{2}(\beta)\right)$ denote the respective inclusion and set $f_{\alpha, \beta}:=\mathrm{H}_{*}\left(i_{\alpha, \beta}\right): A_{\alpha} \rightarrow A_{\beta}$.

Then, the inclusions $i_{\alpha}:\left(N_{1}(\alpha), N_{2}(\alpha)\right) \rightarrow\left(N_{1}, N_{2}\right)$ induce an isomorphism

$$
j: \operatorname{dirlim}\left(\mathrm{H}_{*}\left(N_{1}(\alpha), N_{2}(\alpha)\right), f_{\alpha, \beta}\right) \rightarrow \mathrm{H}_{*}\left[N_{1}, N_{2}\right], \quad[(\alpha, x)] \mapsto \mathrm{H}_{*}\left(p \circ i_{\alpha}\right)(x),
$$

where $p: N_{1} \rightarrow N_{1} / N_{2}$ denotes the canonical projection.

Lemma 3.3. Let $\left(N_{1}, N_{2}\right)$ be an index pair for $\left(y_{0}, K\right)$. Then there are a constant $\tau \in \mathbb{R}^{+}$and a set $N_{2}^{\prime} \subset N_{1}$ such that:

(1) $N_{2} \subset N_{2}^{\prime} \subset N_{2}^{-\tau}$

(2) $\left(N_{1}, N_{2}^{\prime}\right)$ is a regular index pair for $\left(y_{0}, K\right)$.

Proof. By Lemma 4.8 in [7], $N_{2}^{-T}$ is a neighbourhood of $N_{2}$ in $N_{1}$ provided that $T$ is sufficiently large. It follows that $N_{2} \cap \operatorname{cl}\left(N_{1} \backslash N_{2}^{-T}\right)=\emptyset$. By Urysohn's lemma, there exists a continuous function $f: N_{1} \rightarrow[0,1]$ such that $f(x)=0$ on $N_{2}$ and $f(x)=1$ on $\operatorname{cl}\left(N_{1} \backslash N_{2}^{-T}\right)$.

Set

$$
\lambda(x):=\int_{0}^{T(x)} f\left(x \chi_{y_{0}} s\right) \mathrm{d} s,
$$

where $T(x):=\sup \left\{t \in \mathbb{R}^{+}: x \chi_{y_{0}}[0, t] \subset N_{1} \backslash N_{2}\right\}$, in order to guarantee that the integrand is defined.

It is easy to see that $\lambda(x)=0$ on $N_{2}$ and $\lambda(x) \leq T(x)$ for all $x \in N_{1}$. Next, we are going to prove the left-hand inequality of

$$
T(x)-T \leq \lambda(x) \leq T(x) .
$$


One has $\lambda(x) \geq 0$ for all $x \in N_{1}$, so let $x \in N_{1}$ with $T(x)>T$. It follows that $f\left(x \chi_{y_{0}} s\right)=1$ for all $s \in[0, T(x)-T]$, so

$$
T(x)-T=\int_{0}^{T(x)-T} f\left(x \chi_{y_{0}} s\right) d s \leq \lambda(x),
$$

proving (3) for all $x \in N_{1}$.

We need to show that $\lambda$ is continuous. Suppose that $x_{n} \rightarrow x_{0}$ is a sequence and $\lambda\left(x_{0}\right)<\infty$. Assume additionally that $T\left(x_{n}\right)$ is unbounded, so it is possible extract a subsequence $x_{n}^{\prime}$ with $T\left(x_{n}^{\prime}\right) \rightarrow \infty$. We have $x_{n}^{\prime} \chi_{y_{0}} s \in N_{1} \backslash N_{2}^{-T}$ for all $s<T\left(x_{n}^{\prime}\right)-T$ and all $n \in \mathbb{N}$, so $x_{0} \chi_{y_{0}} s \in \operatorname{cl}\left(N_{1} \backslash N_{2}^{-T}\right) \subset N_{1} \backslash N_{2}$ for all $s \in \mathbb{R}^{+}$, which in turn implies that $T\left(x_{0}\right)=\infty$. However by (3), $\lambda\left(x_{0}\right) \geq T\left(x_{0}\right)-T=\infty$, which is a contradiction. Consequently, the sequence $\left(T\left(x_{n}\right)\right)_{n}$ must be bounded.

We further have $x_{n} \chi_{y_{0}}\left[0, T\left(x_{n}\right)\right] \subset N_{1}$ and $x_{n} \chi_{y_{0}} T\left(x_{n}\right) \in N_{2}$ for all $n \in \mathbb{N}$. $T\left(x_{n}\right)$ is bounded, so we may choose a subsequence $x_{n}^{\prime}$ with $T\left(x_{n}^{\prime}\right) \rightarrow t_{0}<\infty$. It follows that

$$
\lambda\left(x_{n}^{\prime}\right) \rightarrow \int_{0}^{t_{0}} f\left(x \chi_{y_{0}} s\right) d s=\lambda\left(x_{0}\right),
$$

where the last equality stems from the facts that $N_{2}$ is positively invariant and $f(x)=0$ on $N_{2}$. This readily implies that $\lambda\left(x_{n}\right) \rightarrow \lambda\left(x_{0}\right)$.

Finally if $\lambda\left(x_{0}\right)=\infty$, then $x_{0} \chi_{y_{0}} s \in N_{1} \backslash N_{2}^{-T}$ for all $s \in \mathbb{R}^{+}$. Arguing by contradiction, assume that there exist a real number $t_{0}$ and a subsequence $x_{n}^{\prime}$ with $\lambda\left(x_{n}^{\prime}\right) \leq t_{0}$ for all $n \in \mathbb{N}$. From (3), one obtains that $x_{n}^{\prime} \chi_{y_{0}} t_{n} \in N_{2}^{-T}$ for some $t_{n} \in\left[0, t_{0}\right]$. Taking subsequences, we may assume w.l.o.g. that $t_{n} \rightarrow t_{0}^{\prime} \leq t_{0}$, so $x_{0} \chi_{y_{0}} t_{0}^{\prime} \in N_{2}^{-T}$, implying that $\lambda\left(x_{0}\right) \leq T\left(x_{0}\right) \leq t_{0}^{\prime}+T$. This is a contradiction and completes the proof that $\lambda$ is continuous.

It is easy to see that $N_{2}^{\prime}:=\lambda^{-1}([0, T+1])$ is a closed neighborhood of $N_{2}^{-T}$ in $N_{1}$. Moreover, $\lambda$ is monotone decreasing along the semiflow, so $\left(N_{1}, N_{2}^{\prime}\right)$ is an index pair.

By (3), it holds that $N_{2}^{\prime} \subset N_{2}^{-\tau}$, where $\tau:=2 T+1$. It follows from Lemma 2.7 in [7] that $\left(N_{1}, N_{2}^{-\tau}\right)$ is an index pair. In conjunction with Lemma 2.8 in [6], one concludes that $\left(N_{1}, N_{2}^{\prime}\right)$ is an index pair for $\left(y_{0}, K\right)$.

Let $x \in N_{1} \backslash N_{2}^{\prime}$ and recall the definition $T_{i}(x):=\sup \left\{t \in \mathbb{R}^{+}: x \chi_{y_{0}}[0, t] \subset\right.$ $\left.N_{1} \backslash N_{2}^{\prime}\right\}$ of the inner exit time. We have $\lambda\left(x \chi_{y_{0}} T_{i}(x)\right)=T+1$ and $f(x)=1$ on $N_{1} \backslash N_{2}^{\prime}$, so $\lambda(x)=T_{i}(x)+T+1$. $\lambda$ is continuous as already proved, so $\left(N_{1}, N_{2}^{\prime}\right)$ is a regular index pair as claimed.

Using regular index pairs, it is easy to prove the following stronger version of Corollary 4.9 in [6].

Lemma 3.4. Let $y_{0} \in Y$ and $K \subset \mathcal{H}^{+}\left(y_{0}\right) \times X$ an isolated invariant set admitting a strongly admissible isolating neighbourhood.

If $\left(N_{1}, N_{2}\right)$ is an index pair for $\left(y_{0}, K\right)$, and $\mathrm{h}\left(N_{1} / N_{2}, N_{2}\right) \neq \overline{0}$, then there are a $t_{0} \in \mathbb{R}^{+}$and a solution $u:\left[t_{0}, \infty\left[\rightarrow N_{1} \backslash N_{2}\right.\right.$ of $\Phi_{y_{0}}$.

Proof. In view of Lemma 3.3, one may assume without loss of generality that $\left(N_{1}, N_{2}\right)$ is a regular index pair. Suppose that $\left(N_{1}, N_{2}\right)$ is such that for every $t_{0} \in \mathbb{R}^{+}$there does not exist a solution $u:\left[t_{0}, \infty\left[\rightarrow N_{1} \backslash N_{2}\right.\right.$ of $\Phi_{y_{0}}$. Then the 
NONAUTONOMOUS CONLEY INDEX THEORY: THE CONNECTING HOMOMORPHISM 11

(continuous) exit time $T_{i}$ satisfies

$$
T_{i}(x)=\sup _{t \in \mathbb{R}^{+}}\left\{x \chi_{y_{0}}[0, t] \subset N_{1} \backslash N_{2}\right\}<\infty \text { for all } x \in N_{1} .
$$

It is easy to see that for each $x \in N_{1}, x \chi_{y_{0}}\left[0, T_{i}(x)\right] \subset N_{1}$ and $x \chi_{y_{0}} T_{i}(x) \in N_{2}$. One can define $H:[0,1] \times N_{1} \rightarrow N_{1}$ by

$$
H(\lambda, x):=x \chi_{y_{0}}\left(\lambda T_{i}(x)\right) .
$$

$H$ is continuous, and $H(\lambda, x)=x$ for all $(\lambda, x) \in[0,1] \times N_{2}$. Consequently, $\left(N_{1} / N_{2}, N_{2}\right)$ and $\left(N_{2} / N_{2}, N_{2}\right)$ are homotopy equivalent, completing the proof because $\mathrm{h}\left(N_{2} / N_{2}, N_{2}\right)=\overline{0}$.

Lemma 3.5. Let $\left(N_{1}, N_{2}\right)$ be a regular index pair with respect to $y_{0} \in Y$. Then the projection $p: N_{1} \rightarrow N_{1} / N_{2}$ induces an isomorphism $p_{*}: \mathrm{H}_{*}\left(N_{1}, N_{2}\right) \rightarrow$ $\mathrm{H}_{*}\left(N_{1} / N_{2}, N_{2} / N_{2}\right)$.

Proof. The (inner) exit time $T_{i}(x):=\sup \left\{t \in \mathbb{R}^{+}: x \chi_{y_{0}}[0, t] \subset N_{1} \backslash N_{2}\right\}$ is continuous. Therefore, $N_{2}^{\prime}:=N_{2}^{-1}=\left\{x \in N_{1}: x \chi_{y_{0}} s \in N_{2}\right.$ for some $\left.s \in[0,1]\right\}$ is a neighbourhood of $N_{2}$ in $N_{1}$. Define $H:[0,1] \times N_{1} \rightarrow N_{1}$ by $H(\lambda, x):=$ $x \chi_{y_{0}}\left(\lambda \min \left\{T_{i}(x), 1\right\}\right)$. Using $H$, we conclude that there are inclusion induced isomorphisms

$$
\begin{aligned}
& \mathrm{H}_{*}\left(N_{1}, N_{2}\right) \rightarrow \mathrm{H}_{*}\left(N_{1}, N_{2}^{\prime}\right) \\
& \mathrm{H}_{*}\left(N_{1} / N_{2},\left\{N_{2}\right\}\right) \rightarrow \mathrm{H}_{*}\left(N_{1} / N_{2}, N_{2}^{\prime} / N_{2}\right) .
\end{aligned}
$$

Using the excision property of homology, it follows that $p$ induces an isomorphism $\mathrm{H}_{*}\left(N_{1}, N_{2}^{\prime}\right) \rightarrow \mathrm{H}_{*}\left(N_{1} / N_{2}, N_{2}^{\prime} / N_{2}\right)$. The proof is complete.

of Lemma 3.2. In view of Lemma 3.5. it is sufficient to consider the inclusion induced mapping

$$
j^{\prime}: \operatorname{dirlim}\left(\mathrm{H}_{*}\left(N_{1}(\alpha), N_{2}(\alpha)\right), \mathrm{H}_{*}\left(i_{\alpha, \beta}\right)\right) \rightarrow \mathrm{H}_{*}\left(N_{1}, N_{2}\right) .
$$

$j^{\prime}$ is an isomorphism since $\mathrm{H}$ is assumed to be a homology theory with compact supports (see e.g. [13, Theorem 13 in Section 4.8]).

Lemma 3.6. Let the direct system $\left(A_{\alpha}, f_{\alpha, \beta}\right)$ be defined as in Lemma 3.2. $a<c$, and $\alpha:=[a, c] \subset[b, c]=: \beta$.

Then, $f_{\alpha, \beta}$ is an isomorphism.

Proof. Let $h>0$ and $\gamma:=[d-h, d] \subset \mathbb{R}^{+}$be an otherwise arbitrary interval. Since $\left(N_{1}, N_{2}\right)$ is assumed to be a regular index pair, the inner exit time $T(x):=\sup \{t \in$ $\left.\mathbb{R}^{+}: x \chi_{y_{0}}[0, t] \subset N_{1} \backslash N_{2}\right\}$ is continuous.

We can define a continuous mapping $H:[0,1] \times N_{1}(\gamma) \rightarrow N_{1}(\gamma)$ by

$$
H(\lambda,(d-t, x)):= \begin{cases}(d-t, x) \chi_{y_{0}}(\lambda t) & \lambda t \leq T(x) \\ (d-t, x) \chi_{y_{0}} T(x) & \lambda t>T(x) .\end{cases}
$$

It follows that $\left(N_{1}^{\prime}(\gamma), N_{2}(\gamma)\right)$ is a strong deformation retract of $\left(N_{1}(\gamma), N_{2}(\gamma)\right)$, where we set

$$
N_{1}^{\prime}:=N_{1}(\{d\}) \cup N_{2} .
$$

Therefore, the inclusion $\left(N_{1}^{\prime}(\gamma), N_{2}(\gamma)\right) \subset\left(N_{1}(\gamma), N_{2}(\gamma)\right)$ defines an isomorphism

$$
\mathrm{H}_{*}\left(N_{1}^{\prime}(\gamma), N_{2}(\gamma)\right) \rightarrow \mathrm{H}_{*}\left(N_{1}(\gamma), N_{2}(\gamma)\right) .
$$


Moreover, the inclusion $\left(N_{1}^{\prime}(\alpha), N_{2}(\alpha)\right) \rightarrow\left(N_{1}^{\prime}(\beta), N_{2}(\beta)\right)$ induces an isomorphism in homology by excision 4 .

Summing up, there is a commutative diagram, where every arrow denotes an isomorphism induced by the inclusion of the respective subspaces:

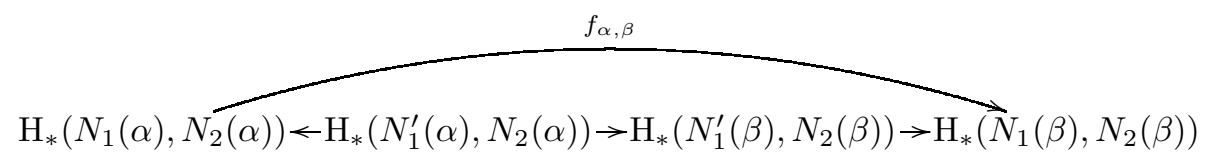

Lemma 3.7. Let $y_{0} \in Y,\left(N_{1}, N_{2}\right)$ be a regular index pair with respect to $y_{0}$ and $\varepsilon>0$ be arbitrary (not necessarily small). Let $\Gamma$ denote the set of all non-empty compact subintervals of $\mathbb{R}^{+}$ordered by inclusion. Let $\alpha, \beta \in \Gamma, N_{2}^{-\varepsilon}:=N_{2}^{-\varepsilon}\left(N_{1}\right)$ and $A_{\alpha}^{\varepsilon}:=\mathrm{H}_{*}\left(N_{1}(\alpha), N_{2}^{-\varepsilon}(\alpha)\right)$. Finally, let $f_{\alpha, \beta}^{\varepsilon}: A_{\alpha}^{\varepsilon} \rightarrow A_{\beta}^{\varepsilon}$ be inclusion induced.

Then for ever $y^{5}$ pair $[a, c] \subset[b, c]$ of subintervals of $\mathbb{R}^{+}$, it holds that $f_{\alpha, \beta}^{\varepsilon}$ is an isomorphism.

Proof. As in the proof of Lemma 3.6, it can be shown that $\left(N_{1}^{\prime}, N_{2}^{\prime}\right):=\left(N_{1}(\{c\}) \cup\right.$ $N_{2}([a, c]), N_{2}^{-\varepsilon}(\{c\}) \cup N_{2}([a, c])$ is a strong deformation retract of $\left(N_{1}[a, c], N_{2}^{-\varepsilon}([a, c])\right)$.

We have $N_{2}^{-\varepsilon}=T^{-1}([0, \varepsilon])$, where $T: N_{1} \rightarrow[0, \infty]$ denotes the inner exit time $T(x):=\sup \left\{t \in \mathbb{R}^{+}: x \chi_{y_{0}}[0, t] \subset N_{1} \backslash N_{2}\right\}$. Due to the continuity of $T$, $N_{2}^{-\varepsilon / 2}\left(N_{1}\right) \cap N_{1}^{\prime}$ is a neighbourhood of $N_{2}$ in $N_{1}^{\prime}$. Hence, $\left(N_{1}(\{c\}), N_{2}^{-\varepsilon}(\{c\})\right) \subset$ $\left(N_{1}^{\prime}, N_{2}^{\prime}\right)$ induces an isomorphism in homology by excision.

Further details are omitted.

Lemma 3.8. Let $\left(N_{1}, N_{2}\right)$ be a regular index pair with respect to $y_{0} \in Y, \varepsilon>0$, and let the direct system $\left(A_{\alpha}^{\varepsilon}, f_{\alpha, \beta}\right)$ be defined as in Lemma 3.7.

Suppose we are given a strictly monotone increasing sequence $\left(a_{n}\right)_{n}$ in $\mathbb{R}^{+}$with $a_{n} \rightarrow \infty$. Define a direct system $\left(B_{k}^{\varepsilon}, g_{k, l}\right)$, where $k, l \in \mathbb{N}, k \leq l$ and $B_{k}^{\varepsilon}:=A_{\left\{a_{k}\right\}}^{\varepsilon}$. Moreover, for $k \leq l$ and in view of Lemma 3.7. we can define

$$
g_{k, l}:=f_{\left\{a_{l}\right\},\left[a_{k}, a_{l}\right]}^{-1} \circ f_{\left\{a_{k}\right\},\left[a_{k}, a_{l}\right]} .
$$

Then, the inclusions $i_{k}:\left(N_{1}\left(\left\{a_{k}\right\}\right), N_{2}^{-\varepsilon}\left(\left\{a_{k}\right\}\right) \rightarrow\left(N_{1}, N_{2}^{-\varepsilon}\right)\right.$ induce an isomorphism

$$
g: \operatorname{dirlim}\left(B_{k}^{\varepsilon}, g_{k, l}\right) \rightarrow \mathrm{H}_{*}\left[N_{1}, N_{2}^{-\varepsilon}\right], \quad[k, x] \mapsto \mathrm{H}_{*}\left(p \circ i_{k}\right)(x),
$$

where $p$ is the canonical projection onto the quotient space as given by Lemma 3.5.

Proof. First of all, we need to prove that $g$ is well defined. Let there be given two representations $[k, x]=\left[l, x^{\prime}\right]$ of the same element in $\operatorname{dirlim}\left(B_{k}^{\varepsilon}, g_{k, l}\right)$, that is,

\footnotetext{
${ }^{4}$ Here, the assumption $a \neq c$ is used.

${ }^{5}$ In contrast to Lemma 3.6. the case $\alpha=\{c\}$ is included.
} 
$g_{k, l}(x)=x^{\prime}$. The following diagram with inclusion induced morphisms is commutative.

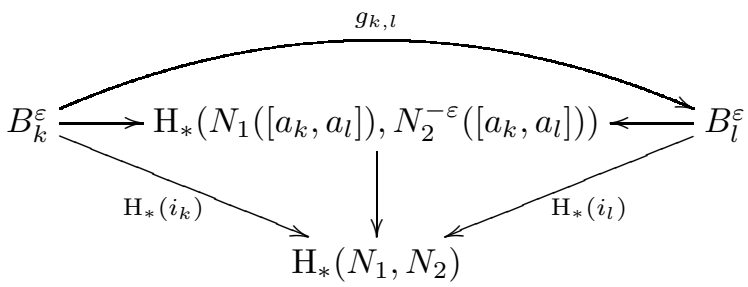

Consequently, $g$ is well defined.

Let the isomorphism $j: \operatorname{dirlim}\left(A_{\alpha}^{\varepsilon}, f_{\alpha, \beta}\right) \rightarrow \mathrm{H}_{*}\left[N_{1}, N_{2}^{-\varepsilon}\right]$ be given by Lemma 3.2 with $\left(N_{1}, N_{2}\right)$ replaced by $\left(N_{1}, N_{2}^{-\varepsilon}\right)$. It is clear that $j\left(\left[a_{k}, x\right]\right)=g([k, x])$ for all $[k, x] \in \operatorname{dirlim}\left(B_{k}^{\varepsilon}, g_{k, l}\right)$. Letting $y \in \mathrm{H}_{*}\left(N_{1}, N_{2}^{-\varepsilon}\right)$, there exists $[\alpha, x] \in$ $\operatorname{dirlim}\left(A_{k}^{\varepsilon}, f_{\alpha, \beta}\right)$ such that $j([\alpha, x])=y$. We can assume without loss of generality that $\alpha=\left[a_{k}, a_{l}\right]$ for $k, l \in \mathbb{N}$ with $k \leq l$. It follows from Lemma 3.7 that $j([\alpha, x])=$ $j\left(\left[\left\{a_{l}\right\}, x^{\prime}\right]\right)=y$ for some $x^{\prime} \in A_{\left\{a_{k}\right\}}^{\varepsilon}=B_{k}^{\varepsilon}$. Thus, $g$ is an epimorphism.

Assume that $g([k, x])=0$. Since $j$ is an isomorphism, it follows that $\left[\left\{a_{k}\right\}, x\right]=$ 0 , so there exists a compact interval $[a, b]$ with $a \leq a_{k} \leq b$ such that $f_{\left\{a_{k}\right\},[a, b]}(x)=$ 0 . We even have $f_{\left\{a_{k}\right\},\left[a, a_{l}\right]}(x)=f_{\left[a_{k}, a_{l}\right],\left[a, a_{l}\right]} \circ f_{\left\{a_{k}\right\},\left[a_{k}, a_{l}\right]}(x)=0$ provided that $b \leq a_{l}$, so $f_{\left\{a_{k}\right\},\left[a_{k}, a_{l}\right]}=0$. From Lemma 3.7 one obtains $g_{k, l}(x)=f_{\left\{a_{l}\right\},\left[a_{k}, a_{l}\right]}^{-1} \circ$ $f_{\left\{a_{k}\right\},\left[a_{k}, a_{l}\right]}(x)=0$, so $[k, x]=\left[l, g_{k, l}(x)\right]=[l, 0]=0$. We have proved that $g$ is a monomorphism.

\section{UNIFORMLY CONNECTED ATTRACTOR-REPELLER DECOMPOSITIONS}

In analogy to the previous section, let $V \subset Y \times X$ and define 6

$$
\begin{aligned}
V(y) & :=\{x:(y, x) \in V\} & \\
V(U) & :=V \cap(U \times X) & \text { where } U \subset Y .
\end{aligned}
$$

Definition 4.1. Let $\left(y_{0}, K, A, R\right)$ be an attractor-repeller decomposition. We say that $A$ and $R$ are not uniformly connected (in $K$ ) if there exists an $y \in \omega\left(y_{0}\right)$, an open neighbourhood $U_{A}$ of $A$ in $\mathcal{H}^{+}\left(y_{0}\right) \times X$ and an open neighbourhood $U_{R}$ of $R$ in $\mathcal{H}^{+}\left(y_{0}\right) \times X$ such that $U_{A} \cap U_{R}=\emptyset$ and $K(y) \subset U_{A}(y) \cup U_{R}(y)$.

Otherwise, $\left(y_{0}, K, A, R\right)$ is called uniformly connected.

The following theorem is the main result of this section, and the rest of the section is devoted to its proof. The strategy is to exploit Lemma 3.8 together with the assumption that $A$ and $R$ are not uniformly connected.

Theorem 4.2. Let $\left(y_{0}, K, A, R\right)$ be an attractor-repeller decomposition, and let there exist a strongly admissible isolating neighbourhood $N \subset \mathcal{H}^{+}\left(y_{0}\right) \times X$ for $K$.

The connecting homomorphism of the associated attractor-repeller sequence is trivial if $\left(y_{0}, K, A, R\right)$ is not uniformly connected.

Lemma 4.3. Let $\left(y_{0}, K, A, R\right)$ be an attractor-repeller decomposition such that $A$ and $R$ are not uniformly connected. Suppose that $K$ is compact $]^{7}$

\footnotetext{
${ }^{6}$ for arbitrary spaces $Y$ and $X$, in particular also $Y=\mathbb{R}^{+}$

${ }^{7}$ This follows from the assumptions of Theorem 4.2
} 
Then there are $y^{\prime} \in \omega\left(y_{0}\right)$, an open neighbourhood $U_{A} \subset \mathcal{H}^{+}\left(y_{0}\right) \times X$ of $A$, an open neighbourhood $U_{R} \subset \mathcal{H}^{+}\left(y_{0}\right) \times X$ of $R$ and a neighbourhood $U$ of $y^{\prime}$ in $\mathcal{H}^{+}\left(y_{0}\right)$ such that $U_{A} \cap U_{R}=\emptyset$ and $K(y) \subset U_{A}(y) \cup U_{R}(y)$ for all $y \in U$.

Proof. Since $A$ and $R$ are not uniformly connected, there must exist a $y^{\prime} \in \omega\left(y_{0}\right)$, an open neighbourhood $U_{A} \subset \mathcal{H}^{+}\left(y_{0}\right) \times X$ of $A$ and an open neighbourhood $U_{R} \subset$ $\mathcal{H}^{+}\left(y_{0}\right) \times X$ of $R$ such that $U_{A} \cap U_{R}=\emptyset$ and $K\left(y^{\prime}\right) \subset U_{A}\left(y^{\prime}\right) \cup U_{R}\left(y^{\prime}\right)$.

Suppose that the lemma does not hold. Then there is a sequence $\left(y_{n}, x_{n}\right) \in K$ such that $y_{n} \rightarrow y^{\prime}$ and $x_{n} \in K\left(y_{n}\right) \backslash\left(U_{A} \cup U_{R}\right)$. Due to the compactness of $K$, we may assume without loss of generality that $\left(y_{n}, x_{n}\right) \rightarrow\left(y^{\prime}, x_{0}\right) \in K$. Thus, $\left(y^{\prime}, x_{0}\right) \in K \backslash\left(U_{A} \cup U_{R}\right)$, which is a contradiction.

Let $y^{\prime} \in \omega\left(y_{0}\right), U \subset \mathcal{H}^{+}\left(y_{0}\right)$ a closed neighbourhood of $y^{\prime}, U_{A} \subset \mathcal{H}^{+}\left(y_{0}\right) \times X$ an open neighbourhood of $A$ and $U_{R} \subset \mathcal{H}^{+}\left(y_{0}\right) \times X$ an open neighbourhood of $R$ for which the conclusions of Lemma 4.3 hold. There is a sequence $t_{n} \rightarrow \infty$ in $\mathbb{R}^{+}$such that $a_{n}:=y_{0}^{t_{n}} \in U$ for all $n \in \mathbb{N}$. By the choice of $U$, one has $U_{A}\left(a_{n}\right) \cap U_{R}\left(a_{n}\right)=\emptyset$ and $K\left(a_{n}\right) \subset U_{A}\left(a_{n}\right) \cup U_{R}\left(a_{n}\right)$ for all $n \in \mathbb{N}$.

Lemma 4.4. Let $N_{\varepsilon}^{\prime}:=\operatorname{cl}_{\mathcal{H}^{+}\left(y_{0}\right) \times X} \bigcup_{(y, x) \in K} B_{\varepsilon}(y, x)$.

There is a real $\varepsilon_{0}>0$ such that for all $\varepsilon<\varepsilon_{0}, N_{\varepsilon}^{\prime} \subset \mathcal{H}^{+}\left(y_{0}\right) \times X$ is an isolating neighbourhood for $K$ such that $N_{\varepsilon}^{\prime}(U) \subset U_{A}(U) \cup U_{R}(U)$.

Proof. It is sufficient to prove that for all $\varepsilon>0$ sufficiently small, $N_{\varepsilon}^{\prime}(U)$ is an isolating neighbourhood for $K$ in $\mathcal{H}^{+}\left(y_{0}\right) \times X$ and $N_{\varepsilon}^{\prime}(U) \subset U_{A} \cup U_{R} . K$ is (by assumption) an isolated invariant set admitting a strongly admissible isolating neighbourhood, so for small $\varepsilon>0, N_{\varepsilon}^{\prime}$ is an isolating neighbourhood for $K$.

Suppose that $N_{\varepsilon}^{\prime}(U) \subset U_{A} \cup U_{R}$ does not hold for small $\varepsilon>0$. Using the compactness of $K$ and the closedness assumption on $U$, one concludes that there is a point $(y, x) \in K(U) \backslash\left(U_{A} \cup U_{R}\right)=\emptyset$, which is a contradiction.

Fix an isolating neighbourhood $N^{\prime} \subset \mathcal{H}^{+}\left(y_{0}\right) \times X$ for $K$ for which the conclusions of Lemma 4.4 hold. Recall that $r: \mathbb{R}^{+} \times X \rightarrow \mathcal{H}^{+}\left(y_{0}\right) \times X$ is defined by $r(t, x):=$ $\left(y_{0}^{t}, x\right)$. By using Lemma 4.3 in [7], one obtains an index triple $\left(N_{1}, N_{2}, N_{3}\right)$ for $\left(y_{0}, K, A, R\right)$ with $N_{1} \subset r^{-1}\left(N^{\prime}\right)$.

Lemma 4.5. Letting $\hat{N}_{\varepsilon}^{\prime}:=r^{-1}\left(N_{\varepsilon}^{\prime}\right)$, there exist reals $\varepsilon>0, T>0$ such that

$$
\hat{N}_{\varepsilon}^{\prime} \backslash N_{2}^{-T}\left(N_{1}\right) \subset r^{-1}\left(U_{R}\right) .
$$

Proof. Suppose to the contrary that there exists sequences $\left(\varepsilon_{n}\right)_{n}$ in $\mathbb{R}^{+}$and $\left(t_{n}, x_{n}\right)$ in $\operatorname{cl}_{\mathbb{R}^{+} \times X}\left(\hat{N}_{\varepsilon_{n}}^{\prime} \backslash N_{2}^{-n}\left(N_{1}\right)\right)$ with $\varepsilon_{n} \rightarrow 0$ and $r\left(t_{n}, x_{n}\right) \notin U_{R}$ for all $n \in \mathbb{N}$. As $\varepsilon_{n} \rightarrow 0$, there is a subsequence $\left(t_{n(k)}, x_{n(k)}\right)_{k}$ such that $r\left(t_{n(k)}, x_{n(k)}\right) \rightarrow(y, x) \in$ $K \backslash U_{R}$.

Let $N_{R}$ be an isolating neighbourhood for $R$ in $\mathcal{H}^{+}\left(y_{0}\right) \times X$ with $N_{1} \backslash N_{2} \subset$ $r^{-1}\left(N_{R}\right)$. We have $(y, x) \pi t \in N_{R}$ for all $t \in \mathbb{R}^{+}$, so $(y, x) \in R \backslash U_{R}=\emptyset$.

Lemma 4.6. For arbitrary $\varepsilon>0$, there is an index triple $\left(L_{1}, L_{2}, L_{3}\right)$ for $\left(y_{0}, K, A, R\right)$ such that $\left(L_{1}, L_{2}, L_{3}\right) \subset\left(\hat{N}_{\varepsilon}^{\prime} \cap N_{1}, N_{2}, N_{3}^{-T}\right)$ for some $T=T(\varepsilon) \in \mathbb{R}^{+}$.

Proof. By choosing $\varepsilon$ smaller if required we can assume without loss of generality that $\hat{N}_{\varepsilon}^{\prime}$ and $N_{3}$ are disjoint. By virtue of Theorem 2.9 in [7, there is an index pair $\left(L_{1}, L_{3}\right)$ for $\left(y_{0}, K\right)$ with $\left(L_{1}, L_{3}\right) \subset\left(\hat{N}_{\varepsilon}^{\prime} \cap N_{1}, N_{3}^{-T}\right)$ for real $T$ sufficiently large. Setting $L_{2}:=L_{1} \cap N_{2}$, it remains to prove that $\left(L_{2}, L_{3}\right)$ is an index pair for $\left(y_{0}, A\right)$. 
(IP2) Let $x \in L_{2}$ and $x \chi t \notin L_{2}$ for some $t>0$. We must have $x \chi t \notin N_{2}$ or $x \chi t \notin L_{1}$. Thus either $x \chi s \in L_{3}$ for some $s \in[0, t]$ or $x \chi[0, t] \subset L_{1}$ and $x \chi s \notin N_{1}$ for some $s \in[0, t]$. The second case leads immediately to a contradiction since $L_{1} \subset N_{1}$.

(IP3) Let $x \in L_{2}$ and $x \chi t \notin L_{2}$ for some $t>0$. Either $x \chi t \notin N_{2}$, so $x \chi s \in$ $\left(\mathbb{R}^{+} \times X\right) \backslash N_{2}$ for some $s \in[0, t]$, or $x \chi t \notin L_{1}$. In the second case, it follows that $x \chi s \in\left(\mathbb{R}^{+} \times X\right) \backslash L_{2}$ for some $s \in[0, t]$.

(IP4) There is an isolating neighbourhood $M^{1}$ (resp. $\left.M^{2}\right)$ for $K$ (resp. $A$ ) with $\mathrm{cl}_{\mathbb{R}^{+} \times X}\left(L_{1} \backslash L_{3}\right) \subset r^{-1}\left(M^{1}\right)\left(\right.$ resp. $\left.\operatorname{cl}_{\mathbb{R}^{+\times X}}\left(N_{2} \backslash N_{3}\right) \subset r^{-1}\left(M^{2}\right)\right)$. Another isolating neighbourhood for $A$ is $M^{3}:=M^{1} \cap M^{2}$. One has $\operatorname{cl}_{\mathbb{R}^{+} \times X}\left(L_{2} \backslash\right.$ $\left.L_{3}\right) \subset \mathrm{cl}_{\mathbb{R}^{+} \times X}\left(L_{1} \backslash L_{3}\right) \subset r^{-1}\left(M^{1}\right)$ and, using the fact that $L_{2}$ and $N_{3}$ are disjoint, $\mathrm{cl}_{\mathbb{R}^{+} \times X}\left(L_{2} \backslash L_{3}\right) \subset \operatorname{cl}_{\mathbb{R}^{+} \times X}\left(N_{2} \backslash N_{3}\right) \subset r^{-1}\left(M^{2}\right)$. Combining the inclusions yields $\operatorname{cl}_{\mathbb{R}^{+} \times X}\left(L_{2} \backslash L_{3}\right) \subset r^{-1}\left(M^{1}\right) \cap r^{-1}\left(M^{2}\right)=r^{-1}\left(M^{1} \cap M^{2}\right)$.

(IP5) There is a neighbourhood $U^{1}$ (resp. $\left.U^{2}\right)$ of $K$ (resp. $A$ ) such that $r^{-1}\left(U^{1}\right) \subset$ $L_{1} \backslash L_{3}$ (resp. $\left.r^{-1}\left(U^{2}\right) \subset N_{2} \backslash N_{3} \subset N_{2}\right) . U^{3}:=U^{1} \cap U^{2}$ is a neighbourhood of $A$ and $r^{-1}\left(U^{3}\right) \subset\left(L_{1} \backslash L_{3}\right) \cap N_{2}$.

By redefining $\left(N_{1}, N_{2}, N_{3}\right)$ and using Lemma 4.6 together with Lemma 4.4 Lemma 4.5 and Lemma 4.5 in [7, one can assume that $\left(N_{1}, N_{2}, N_{3}\right)$ is an index triple for $\left(y_{0}, K, A, R\right)$ such that

$$
\begin{aligned}
N_{1}\left(r^{-1}(U)\right) & \subset r^{-1}\left(U_{A}\right) \cap r^{-1}\left(U_{R}\right) \\
N_{1} \backslash N_{2} & \subset r^{-1}\left(U_{R}\right)
\end{aligned}
$$

and thus also

$$
N_{1} \cap r^{-1}\left(U_{A}\right) \subset N_{2}
$$

Lemma 4.7. Let $\left(N_{1}, N_{2}, N_{3}\right)$ be an index triple for $\left(y_{0}, K, A, R\right)$. Then there is a set $N_{3}^{\prime} \supset N_{3}$ such that $\left(N_{1}, N_{3}^{\prime}\right)$ is a regular index pair for $\left(y_{0}, K\right), N_{3}^{\prime} \subset N_{3}^{-\tau}\left(N_{1}\right)$ for some $\tau \geq 0$ and $\left(N_{2}^{-\tau}\left(N_{1}\right), N_{3}^{\prime}\right)$ is a regular index pair for $\left(y_{0}, A\right)$.

Proof. It follows from Lemma 3.3 that there exist a set $N_{3}^{\prime} \supset N_{3}$ and a constant $\tau \in \mathbb{R}^{+}$such that $\left(N_{1}, N_{3}^{\prime}\right)$ is a regular index pair for $\left(y_{0}, K\right)$ and $N_{3} \subset N_{3}^{\prime} \subset$ $N_{3}^{-\tau}\left(N_{1}\right)$. This means in particular that the (inner) exit time $T: N_{1} \rightarrow[0, \infty]$, $T(x):=\sup \left\{t \in \mathbb{R}^{+}: x \chi_{y_{0}}[0, t] \subset N_{1} \backslash N_{3}^{\prime}\right\}$ is continuous.

One needs to prove that $\left(N_{2}^{-\tau}\left(N_{1}\right), N_{3}^{\prime}\right)$ is an index pair.

(IP2) Let $x \in N_{2}^{-\tau}\left(N_{1}\right)$ and $x \chi_{y_{0}} t \notin N_{2}^{-\tau}\left(N_{1}\right)$ for some $t \geq 0$. One cannot have $x \chi_{y_{0}}[0, t] \subset N_{1}$, so $x \chi_{y_{0}} s \in N_{3}^{\prime}$ for some $s \leq t$.

(IP3) Let $x \in N_{3}^{\prime}$ and $x \chi_{y_{0}} t \notin N_{3}^{\prime}$. It follows that $x \chi_{y_{0}} s \in\left(\mathbb{R}^{+} \times X\right) \backslash N_{1} \subset$ $\left(\mathbb{R}^{+} \times X\right) \backslash N_{2}^{-\tau}\left(N_{1}\right)$ for some $\left.\left.s \in\right] 0, t\right]$ because $\left(N_{1}, N_{3}^{\prime}\right)$ is an index pair.

By Lemma 4.5 in [7, $\left(N_{2}^{-\tau}\left(N_{1}\right), N_{3}\right)$ and $\left(N_{2}^{-\tau}\left(N_{1}\right), N_{3}^{-\tau}\left(N_{1}\right)\right)$ are index pairs for $\left(y_{0}, A\right)$. By using the sandwich lemma [7, Lemma 2.8], one concludes that $\left(N_{2}^{-\tau}\left(N_{1}\right), N_{3}^{\prime}\right)$ is an index pair for $\left(y_{0}, A\right)$.

Finally, the exit time with respect to the index pair $\left(N_{2}^{-\tau}\left(N_{1}\right), N_{3}^{\prime}\right)$ is the restriction of $T$ to $N_{2}^{-\tau}\left(N_{1}\right)$ and therefore continuous that is, the index pair is regular. 
Having proved Lemma4.7 we can assume without loss of generality that $\left(N_{1}, N_{2}, N_{3}\right)$ is an index triple, satisfies (5), (6) , and $\left(N_{1}, N_{3}\right)$ as well as $\left(N_{2}, N_{3}\right)$ are regular index pairs 8 .

By using [7, Theorem 2.9], we can further assume that there exists an index pair $\left(L_{1}, L_{2}\right)$ for $\left(y_{0}, A\right)$ such that $L_{1} \subset r^{-1}\left(U_{A}\right)$.

We are now in a position to complete the proof of Theorem 4.2, In view of the hypotheses of Lemma 3.8, one chooses a small real $\varepsilon>0$, which is unrelated to the previous uses of this letter.

Let $\iota: \mathrm{H}_{*}\left[L_{1}, L_{2}\right] \rightarrow \mathrm{H}_{*}\left[N_{2}^{-\varepsilon}, N_{3}^{-\varepsilon}\right]$ be inclusion induced. It is clear that $\iota$ is an isomorphism. Moreover, the following long exact sequence

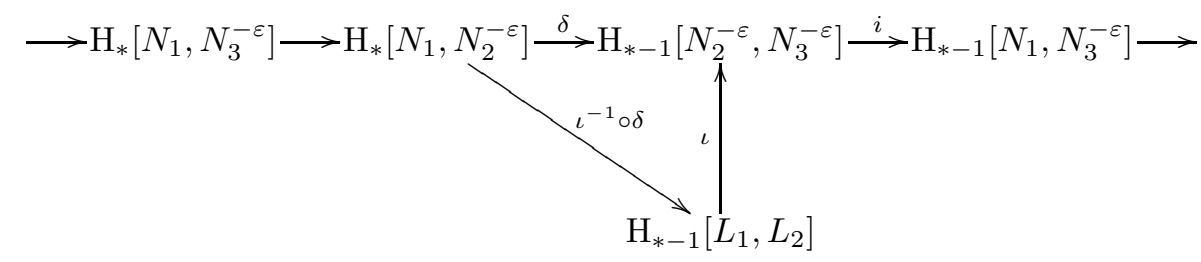

associated with the index triple $\left(N_{1}, N_{2}^{-\varepsilon}, N_{3}^{-\varepsilon}\right)$ gives rise to the connecting homomorphism. The above sequence is exact, so in order to prove $\delta=0$ it is sufficient to prove that $i \circ \iota$ and thus also $i$ is a monomorphism, where $i: \mathrm{H}_{*}\left[N_{2}^{-\varepsilon}, N_{3}^{-\varepsilon}\right] \rightarrow$ $\mathrm{H}_{*}\left[N_{1}, N_{3}^{-\varepsilon}\right]$ is inclusion induced.

Let $\left(a_{n}\right)_{n}$ be a sequence of reals such that $a_{n} \rightarrow \infty$ and $y_{0}^{a_{n}} \in U$ for all $n \in \mathbb{N}$. By using Lemma 3.8, a commutative diagram

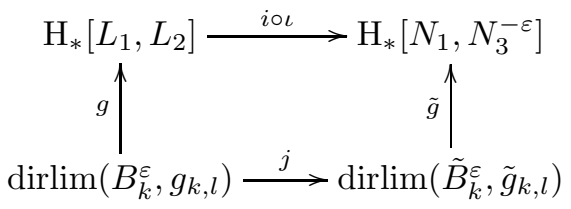

is obtained. Recall that by definition $B_{k}^{\varepsilon}:=\mathrm{H}_{*}\left(L_{1}\left(\left\{a_{k}\right\}\right), L_{3}\left(\left\{a_{k}\right\}\right)\right.$ and $\tilde{B}_{k}^{\varepsilon}:=$ $\mathrm{H}_{*}\left(N_{1}\left(\left\{a_{k}\right\}\right), N_{3}^{-\varepsilon}\left(\left\{a_{k}\right\}\right)\right)$. Let $j_{k}: B_{k}^{\varepsilon} \rightarrow \tilde{B}_{k}^{\varepsilon}$ be inclusion induced and set $j([k, x]):=\left[k, j_{k} k(x)\right]$ for $x \in B_{k}^{\varepsilon}$. We omit the proof that $j$ is well-defined.

By Lemma 3.8, $g$ and $\tilde{g}$ are isomorphisms. Thus it is sufficient to prove that $j$ is a monomorphism. Suppose $j\left(\left[k_{0}, x\right]\right)=\left[k_{0}, j_{k_{0}}(x]\right)=\left[k_{0}, y\right]=0$ for $x \in B_{k_{0}}^{\varepsilon}$, $y \in \tilde{B}_{k_{0}}^{\varepsilon}$ and $k_{0} \in N$. There is an $l \in \mathbb{N}, l \geq k_{0}$ such that $\tilde{g}_{k_{0}, l}(y)=0$. Furthermore, $j_{l} \circ g_{k_{0}, l}(x)=\tilde{g}_{k_{0}, l}(y)=0$. We can hence assume without loss of generality that $j_{k_{0}}(x)=0$.

For brevity, a couple of notational shortcuts are introduced: $M_{1}:=N_{1}\left(\left\{a_{k_{0}}\right\}\right)$, $M_{2}:=N_{2}^{-\varepsilon}\left(\left\{a_{k_{0}}\right\}\right), M_{3}:=N_{3}^{-\varepsilon}\left(\left\{a_{k_{0}}\right\}\right), \hat{U}_{A}:=r^{-1}\left(U_{A}\right)$ and $\hat{U}_{R}:=r^{-1}\left(U_{R}\right)$. It follows from (5) and the choice of the sequence $a_{k}$ that $\mathrm{H}_{*}\left(M_{1} \cap \hat{U}_{A}, M_{3} \cap \hat{U}_{A}\right) \oplus$ $\mathrm{H}_{*}\left(M_{1} \cap \hat{U}_{R}, M_{3} \cap \hat{U}_{R}\right) \simeq \mathrm{H}_{*}\left(M_{1}, M_{3}\right)$. Let the projection $p: \mathrm{H}_{*}\left(M_{1}, M_{3}\right) \rightarrow$ $\mathrm{H}_{*}\left(M_{1} \cap \hat{U}_{A}, M_{3} \cap \hat{U}_{A}\right)$ be defined by the above direct sum decomposition.

${ }^{8}$ One could say that $\left(N_{1}, N_{2}, N_{3}\right)$ is a regular index triple. 
We obtain once again a commutative diagram with inclusion induced homomorphisms and the projection $p$ introduced above.

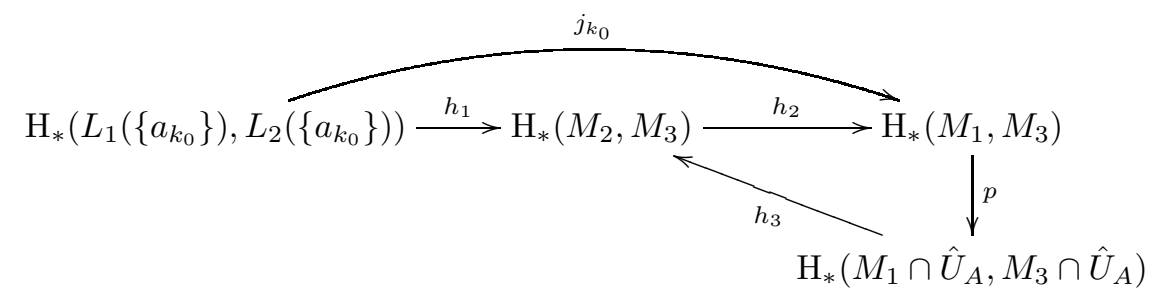

It follows that $h_{1}(x)=h_{3} \circ p \circ h_{2} \circ h_{1}(x)=0$. Moreover, $h_{1}(x)=\iota(g([k, x]))$, implying that $[k, x]=0$ in $\operatorname{dirlim}\left(B_{k}^{\varepsilon}, g_{k, l}\right)$ since $\iota$ and $g$ are isomorphisms. The proof of Theorem 4.2 is complete.

\section{REFERENCES}

[1] P. Brunovsky and P. Polacik, The Morse-Smale structure of a generic reaction-diffusion equation in higher space dimensions, J. Differential Equations 135 (1997), 129-181.

[2] M. C. Carbinatto and K. P. Rybakowski, Homology index braids in infinite-dimensional Conley index theory, Topological Methods Nonlinear Anal. 26 (2005), 35-74.

[3] D. Henry, Geometric theory of semilinear parabolic equations., Lecture Notes in Mathematics. 840. Berlin-Heidelberg-New York: Springer-Verlag. IV, 348 p., 1981.

[4] A. Jänig, Nonautonomous Conley index theory: Continuation of Morse decompositions, Topological Methods Nonlinear Anal. (accepted).

[5] _ The Conley index along heteroclinic solutions of reaction-diffusion equations, J. Differential Equations 252 (2012), 4410-4454.

[6] - A non-autonomous Conley index, Journal of Fixed Point Theory and Applications 19 (2017), 1825-1870.

[7] L Nonautonomous Conley index theory: The homology index and attractor-repeller decompositions, Topological Methods Nonlinear Anal. (accepted).

[8] M. Mrozek and K. P. Rybakowski, A cohomological Conley index for maps on metric spaces, J. Differential Equations 90 (1991), 143-171.

[9] A. Pazy, Semigroups of linear operators and applications to partial differential equations, Springer-Verlag New York, 1983

[10] K. P. Rybakowski, The homotopy index and partial differential equations, Springer, 1987.

[11] G. R. Sell and Y. You, Dynamics of evolutionary equations., Applied Mathematical Sciences 143. New York, NY: Springer. xiii, 670 p., 2002.

[12] George R Sell, Topological dynamics and ordinary differential equations, Van Nostrand Reinhold, 1971.

[13] E. H. Spanier, Algebraic topology, McGraw-Hill, New York, 1966.

Axel Jänig, Institut für Mathematik, Universität Rostock, 18051 Rostock, Germany

E-mail address: axel.jaenig@uni-rostock.de 